\title{
Depression and anxiety in patients with glaucoma: a prospective,
} case-control study

\author{
Charalampos Mitsonis*1, Nikolaos Dimopoulos ${ }^{1}$, Vasiliki Psarra ${ }^{1}$, \\ Zoe Santa ${ }^{1}$, Constantinos Letsas ${ }^{2}$ and Michail Mitsonis ${ }^{3}$
}

\author{
Address: ${ }^{2}$ Psychiatric Hospital of Attica "Dafni", Greece, 2"Evangelismos" General Hospital of Athens, Greece and 3"Tzanio" General Hospital of \\ Piraeus, Greece \\ * Corresponding author
}

\author{
from International Society on Brain and Behaviour: 2nd International Congress on Brain and Behaviour \\ Thessaloniki, Greece. 17-20 November 2005 \\ Published: 28 February 2006 \\ Annals of General Psychiatry 2006, 5(Suppl I):S316 doi:I0.II86/I744-859X-5-SI-S3I6
}

\section{Background}

Many somatic diseases can cause anxiety and depression. The magnitude of the restriction these disorders impose on everyday life and how fatal they are perceived to be, are associated with anxiety and/ or depression. Glaucoma is a serious ocular disease potentially leading to blindness.

The aim of the study was to determine whether patients with glaucoma have more anxiety and/or depressive symptoms than glaucoma-free patients and those with another chronic disease.

\section{Materials and methods}

The study population consisted of three groups: $A=95$ patients with open-angle glaucoma, $B=101$ patients with coronary heart disease, $\mathrm{C}=100$ healthy controls. Subjects of the three groups did not differ significantly in age, gender and education. Patients had no history of any clinically relevant psychiatric disease or systematic use of psychotropic drugs. All subjects were assessed using the Hamilton Rating Scale for Anxiety (HAM-A) and the 17item Hamilton Rating Scale for Depression (HAM-D17).

\section{Results}

A linear relation was observed between anxiety and depression in groups A and B (Pearson correlation, R1 = 0.81 and R2 $=0.78$, respectively). Anxiety and depression levels were significantly higher in A and B group than those in healthy controls $(\mathrm{p}<0.05)$. Anxiety and depression scores in patients with glaucoma did not differ significantly from the scores in patients with coronary disease $(p>0.05)$. In glaucoma patients, anxiety and depression levels were not influenced by visual acuity $(p>0.05)$, vis- ual field severity ( $p>0.05)$, and use of topical beta- blockers $(p>0.05)$.

\section{Discussion}

Glaucoma is positively associated with anxiety and depression. Patients with glaucoma do not report being more anxious or depressed than individuals with another chronic illness (coronary artery disease). 